\title{
Migração intrapélvica do parafuso cefálico da haste intramedular longa para fêmur proximal: Relato de caso*
}

\section{Intrapelvic Migration of the Cephalic Screw of a Proximal Femoral Intramedullary Long Nail: Case Report}

\author{
Elton Luiz Batista Cavalcante ${ }^{1}$ Fernando Junqueira de Faria ${ }^{1}$ Rubens Azevedo Rodrigues ${ }^{2}$ \\ José Eduardo Grandi Ribeiro Filho ${ }^{3}$ Saulo Gomes de Oliveira ${ }^{30}$ Nelson Elias ${ }^{1}$ \\ ${ }^{1}$ Serviço de Ortopedia e Traumatologia, Centro Médico Hospitalar \\ Vila Velha (CMHVV), Vila Velha, ES, Brasil \\ 2 Serviço de Ortopedia e Traumatologia, Hospital Estadual Jayme dos \\ Santos Neves (HEJSN), Serra, ES, Brasil \\ ${ }^{3}$ Departamento de Ortopedia e Traumatologia, Hospital de Vila \\ Velha, Vila Velha, ES, Brasil \\ Endereço para correspondência Saulo Gomes de Oliveira, Master, \\ Departamento de Medicina Esportiva, Universidade Federal do \\ Espírito Santo (UFES), R. Moema, s/n, Quadra 41, Divino Espírito \\ Santo, Vila Velha, ES, 29107-250, Brasil \\ (e-mail: saulo.goliveira@hotmail.com).
}

Rev Bras Ortop 2020;55(1):121-124.

\section{Resumo \\ Palavras-chave \\ - fraturas do fêmur \\ - fixação interna de fraturas \\ - próteses e implantes}

O uso dos dispositivos cefalomedulares tem ganhado popularidade no tratamento das fraturas do fêmur proximal. Apesar das vantagens biomecânicas, várias complicações são descritas, entre as quais a migração medial do parafuso cefálico é pouco conhecida. Os autores apresentam um caso dessa complicação incomum em um implante de haste longa tratada em dois tempos cirúrgicos para a retirada dos implantes e posterior osteossíntese com placa bloqueada para fêmur proximal, assim como o desfecho até a consolidação da fratura e resolução do caso.

The use of cephalomedullary devices has gained popularity in the treatment of proximal femoral fractures. Despite their biomechanical advantages, several complications are well described in the literature. One of these complications, which is rarely reported, is the medial migration of the cephalic screw. The authors present this unusual complication in a long-nail implant, which was treated with removal of the implants as a first step and posterior osteosynthesis with a locked proximal femur plate as a second step, as well as the outcome until fracture consolidation and resolution of the case.

\section{Introdução}

A fratura intertrocantérica do fêmur proximal é uma das mais comuns em pacientes acima de 65 anos, e é frequentemente associada à osteoporose. ${ }^{1}$

Trabalho desenvolvido no Serviço de Ortopedia e Traumatologia, Centro Médico Hospitalar Vila Velha (CMHVV), Vila Velha, ES, Brasil. Originalmente Publicado por Elsevier Editora Ltda.

recebido

10 de Novembro de 2017 aceito

07 de Dezembro de 2017
DOI https://doi.org/

10.1016/j.rbo.2017.12.004. ISSN 0102-3616.
A osteossíntese cefalomedular é o tratamento preferido para as fraturas instáveis por suas vantagens biomecânicas. Apesar disso, várias complicações são descritas, e a soltura lateral e superior do parafuso da cabeça femoral é um dos problemas mais frequentes, que ocorre em $3 \%$ a $10 \%$ dos casos. ${ }^{1}$

Por sua vez, a migração medial do parafuso cefálico é uma complicação pouco frequente, com poucos casos descritos na literatura ortopédica, apesar do alto potencial de morbidade e mortalidade. ${ }^{2}$
Copyright $\odot 2020$ by Sociedade Brasileira License terms de Ortopedia e Traumatologia. Published by Thieme Revinter Publicações Ltda, Rio de Janeiro, Brazil 


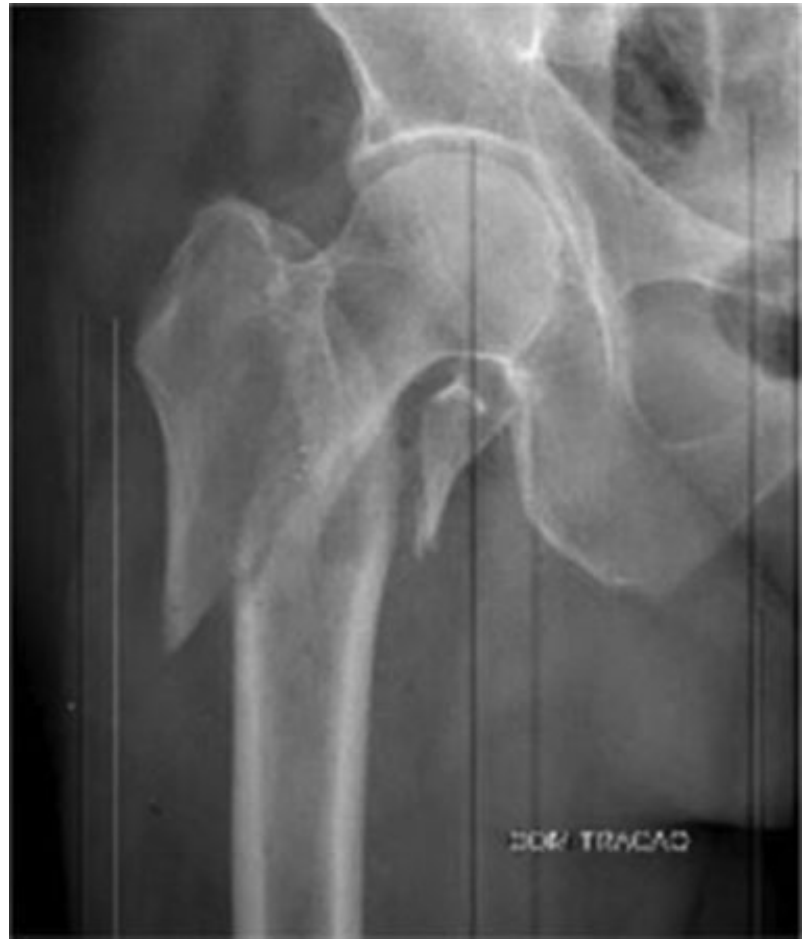

Fig. 1 Radiografia na incidência anteroposterior (AP) do quadril direito (pré-operatório).

Descrevemos uma complicação rara, a migração medial do parafuso cefálico da haste intramedular Gamma 3 (Stryker, Kalamazoo, MI, EUA), registrada três meses após a osteossíntese, bem como a condução do caso até a sua consolidação.

\section{Relato do Caso}

Paciente do sexo feminino, de 70 anos, portadora de hipotireoidismo e osteopenia, admitida com histórico de quedas da própria altura. Ela apresentava dor, encurtamento e deformidade em rotação externa do membro inferior direito associados a incapacidade de deambular. Radiografias da bacia e do quadril direito evidenciaram fratura intertrocantérica (AO 31-A3) da extremidade proximal do fêmur direito (-Fig. 1).

A paciente foi submetida a tratamento cirúrgico sem intercorrências, com o princípio da estabilidade relativa, com redução indireta na mesa de tração esquelética e osteossíntese cefalomedular com haste Gamma3 longa de 130 graus, com o auxílio de fluoroscopia intraoperatória.

Na avaliação radiográfica pós-operatória, foi observada uma distância ponta-ápice (DPA) de $26,5 \mathrm{~mm}$ (-Fig. 2). A paciente apresentou condições clínicas favoráveis de alta hospitalar no quarto dia, com prescrição de reabilitação fisioterápica domiciliar e descarga de peso parcial e progressiva no membro inferior direito, conforme tolerância. Pela boa evolução clínica, ela abandonou o auxílio do andador de quatro apoios por volta da sexta semana, mas, após três meses da cirurgia, iniciou quadro de dor súbita no quadril direito sem novo traumatismo ou queda, piorou progressivamente, e evoluiu para bloqueio da articulação coxofemoral após dois dias.

Na reavaliação, notou-se retardo de consolidação da fratura associada a migração medial do parafuso cefálico da haste para a região pélvica ( - Fig. 3 ), sem sinais de irritação peritoneal ao exame físico.

A paciente foi reinternada e, após cinco dias, submetida a laparoscopia exploratória com identificação do parafuso na região retroperitoneal, próximo aos vasos ilíacos internos, sem evidências de agravos a órgãos ou estruturas intraabdominais ou pélvicas (-Fig. 4), o que possibilitou a retirada dos implantes metálicos por via de acesso lateral.

Após uma semana, a paciente foi submetida a nova osteossíntese com redução direta da fratura e fixação com princípio de estabilidade relativa, com placa bloqueada para fêmur proximal (DePuy Synthes, Raynham, MA, EUA) associada a enxerto ósseo esponjoso do ilíaco (-Fig. 5).
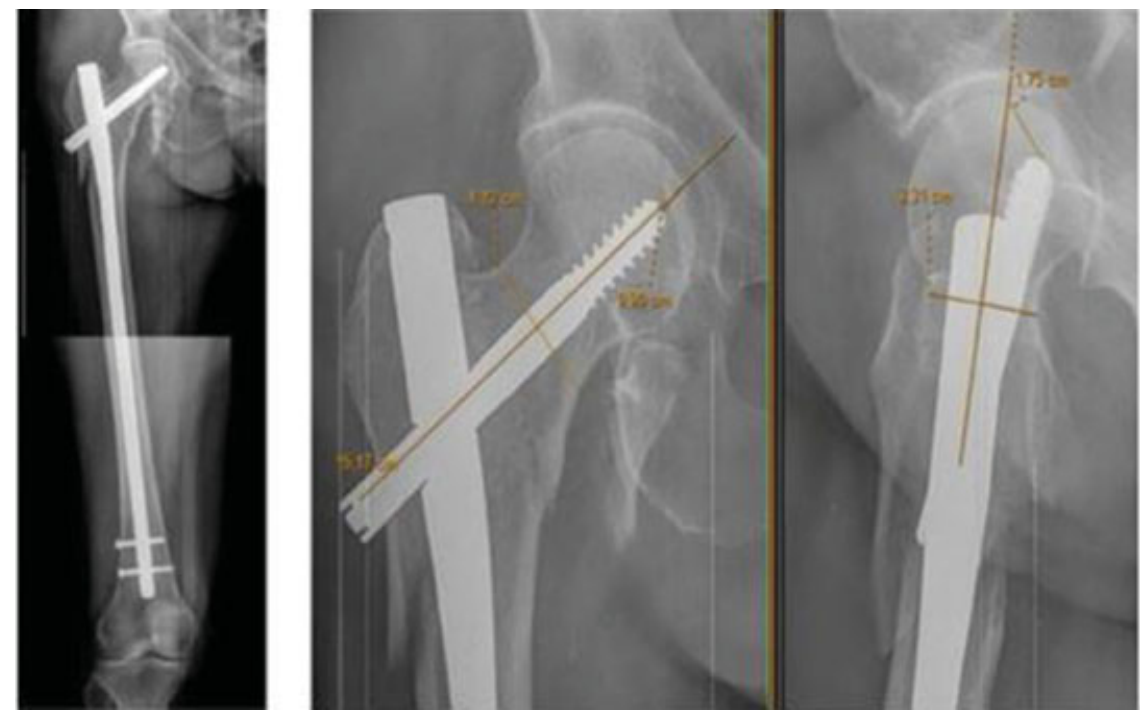

Fig. 2 Radiografias na incidência AP do fêmur direito + AP e perfil do quadril direito demostrando o cálculo da distância ponta-ápice (DPA). 


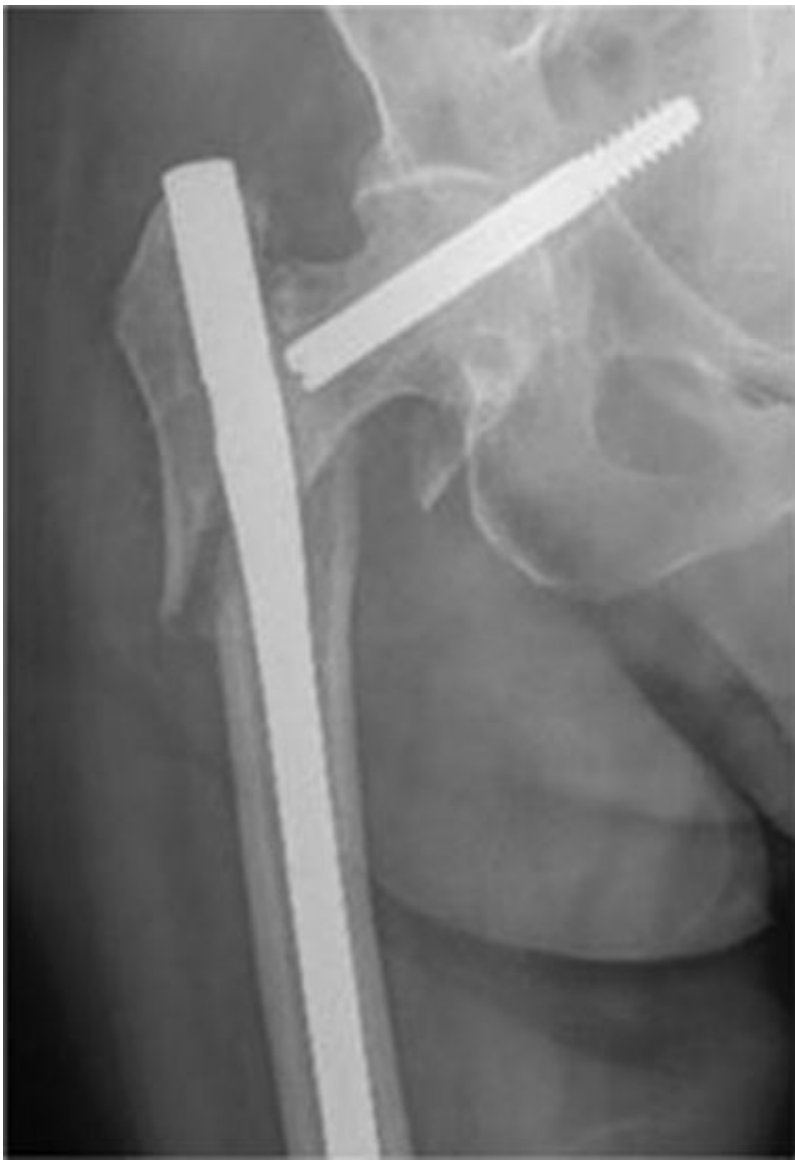

Fig. 3 Radiografia na incidência AP do quadril direito evidenciando a migração medial do parafuso cefálico.

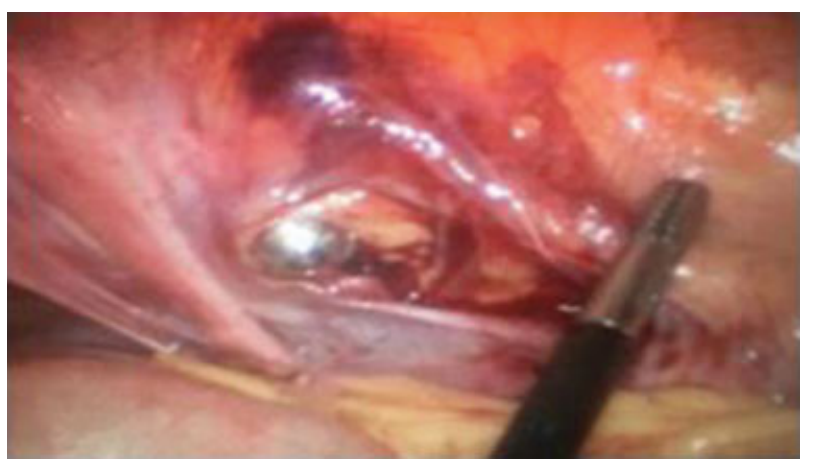

Fig. 4 Imagem videolaparoscópica evidenciando o parafuso cefálico próximo aos vasos ilíacos internos.

A paciente apresentou evolução satisfatória no pós-operatório, e recebeu alta hospitalar após quatro dias, quando estava em boas condições de reiniciar o processo de reabilitação com carga parcial (15\% do peso corporal). Após oito semanas, evidenciou-se consolidação da fratura, que permitiu liberação de carga total.

\section{Discussão}

Na revisão da literatura, não se identificou a complicação de migração medial do parafuso cefálico em implantes de haste longa, dado que todos os relatos encontrados foram asso- ciados a hastes femorais curtas. Tampouco foi encontrado o tratamento dessa complicação com placa bloqueada para fêmur proximal.

Tauber e Resch ${ }^{3}$ relataram um caso de migração intrapélvica do parafuso cefálico com perfuração da porção sigmoide do intestino em uma paciente submetida posteriormente a artroplastia total de quadril (ATQ) cimentada com haste femoral de revisão longa. Flint et $\mathrm{al}^{4}$ relataram um caso de migração intrapélvica em uma paciente de 82 anos; 7 meses após a fixação de uma fratura pertrocantérica instável, ela foi submetida a ATQ não cimentada.

Heineman et $\mathrm{al}^{5}$ descrevem o caso de uma mulher de 83 anos que, após 3 semanas de fixação, apresentou migração do pino para a região pélvica, e foi submetida ao tratamento em dois estágios: inicialmente, retirada dos implantes e, posteriormente ATQ não cimentada. Takasago et $\mathrm{al}^{6}$ apresentaram um caso de uma mulher de 63 anos com migração medial após 6 semanas da cirurgia. Fizeram revisão também em dois estágios, com retirada dos implantes e subsequente ATQ.

Outro relato de migração foi feito por Thein et al $^{7}$ em um paciente com cinco semanas de osteossíntese, o qual foi submetido a embolização da artéria ilíaca interna, pelo contato evidenciado na tomografia computadorizada (TC), e, posteriormente, ATQ. Li et al $^{2}$ descreveram um caso de uma mulher de 77 anos tratada com implante cefalomedular curto deslocado medialmente na décima semana de pósoperatório. Foi submetida a revisão da osteossíntese, com colocação de um pino cefálico mais curto associado a um parafuso canulado para osso esponjoso anterior e paralelamente. Lucke et $\mathrm{al}^{8}$ também descreveram dois casos de migração medial tratados com artroplastia parcial do quadril bipolar.

Mais recentemente, Pinheiro et $\mathrm{al}^{9}$ descreveram a mesma complicação em uma paciente de 92 anos após 5 semanas de osteossíntese com haste cefalomedular curta. Foi submetida a revisão com placa e parafuso deslizante, e evoluiu com cut-out observado aos seis meses de pós-operatório que não foi reabordado por recusa da paciente e de seus parentes.

Diversas causas têm sido postuladas na tentativa de justificar essa complicação potencialmente grave. Weil et al $^{10}$ testaram cinco implantes cefalomedulares, e criaram um modelo de simulação biomecânica. Identificaram que certas condições precisariam estar presentes para alcançar a migração medial, como deficiência no apoio lateral e córtex medial instável, atrito constante dentro da cabeça femoral, e carga axial em varo. Os autores sugerem ainda que o fenômeno parece apresentar risco maior de ocorrer em fraturas instáveis. Outras possíveis causas também relatadas na literatura são: mau posicionamento do parafuso cefálico, inserção inadequada do dispositivo de travamento antirrotatório, DPA aumentada, danos à cabeça femoral por fresagens repetitivas, e necrose avascular da cabeça femoral. $^{6}$

A migração medial do parafuso cefálico é uma complicação pouco frequente na literatura ortopédica, sem descrição de casos encontrados que a associem à haste cefalomedular longa nem ao tratamento com placa bloqueada para fêmur proximal. 
124 Migração intrapélvica do parafuso cefálico da haste intramedular longa Cavalcante et al.
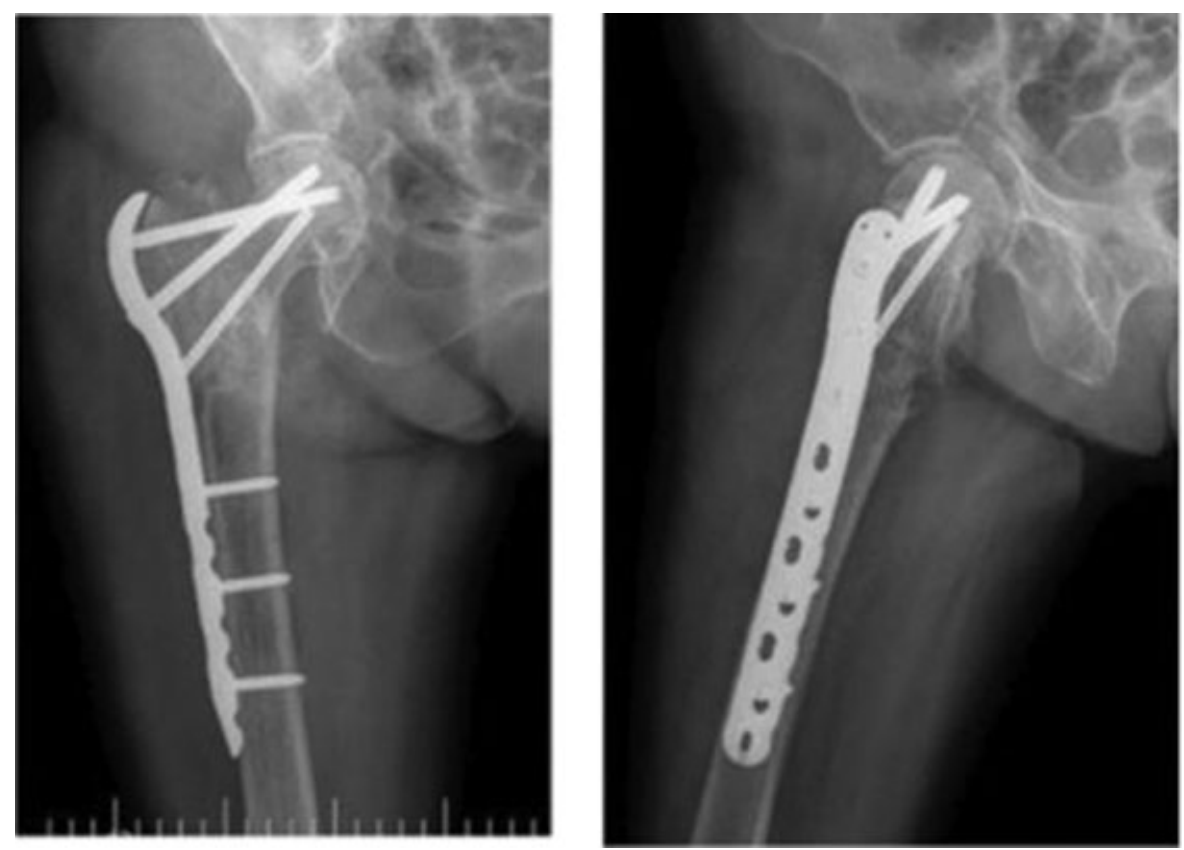

Fig. 5 Radiografias nas incidências AP e de perfil do quadril direito após tratamento definitivo.

Conflito de Interesses

Os autores declaram não haver conflito de interesses.

\section{Referências}

1 Campbell WC, Canale ST, Beaty JH. Campbell's operative orthopaedics. 11th ed. Philadelphia: Mosby/Elsevier; 2008

2 Li X, Heffernan MJ, Kane C, Leclair W. Medial pelvic migration of the lag screw in a short gamma nail after hip fracture fixation: a case report and review of the literature. J Orthop Surg Res 2010; $5: 62$

3 Tauber M, Resch H. Sigmoid perforation after medial migration of lag screw in gamma nailing. Arch Orthop Trauma Surg 2006;126 (02):118-122

4 Flint JH, Sanchez-Navarro CF, Buckwalter JA, Marsh JL. Intrapelvic migration of a gamma nail lag screw: review of the possible mechanisms. Orthopedics 2010;33(04):
5 Heineman DJ, van Buijtenen JM, Heuff G, Derksen EJ, Pöll RG. Intra-abdominal migration of a lag screw in gamma nailing: report of a case. J Orthop Trauma 2010;24(12):e119-e122

6 Takasago T, GotoT, Toki S, et al. Intrapelvic migration of the lag screw in intramedullary nailing. Case Rep Orthop 2014;2014:519045

7 Thein E, De Cannière A, Burn A, Borens O. Medial migration of lag screw after gamma nailing. Injury 2014;45(08):1275-1279

8 Lucke M, Burghardt RD, Siebenlist S, Ganslmeier A, Stöckle U. Medial migration of lag screw with intrapelvic dislocation in gamma nailing-a unique problem? A report of 2 cases. J Orthop Trauma 2010;24(02):e6-e11

9 Pinheiro AC, Alpoim B, Félix A, Alves C, Sousa C, Rodrigues A. Medial migration of the intramedullary Gamma 3 nail - a case report. Rev Bras Ortop 2016;51(06):720-724

10 Weil YA, Gardner MJ, Mikhail G, Pierson G, Helfet DL, Lorich DG. Medial migration of intramedullary hip fixation devices: a biomechanical analysis. Arch Orthop Trauma Surg 2008;128(02):227-234 